\title{
Alien Mindscapes-A Perspective on the Search for Extraterrestrial Intelligence
}

\author{
Nathalie A. Cabrol
}

\begin{abstract}
Advances in planetary and space sciences, astrobiology, and life and cognitive sciences, combined with developments in communication theory, bioneural computing, machine learning, and big data analysis, create new opportunities to explore the probabilistic nature of alien life. Brought together in a multidisciplinary approach, they have the potential to support an integrated and expanded Search for Extraterrestrial Intelligence (SETI ${ }^{1}$ ), a search that includes looking for life as we do not know it. This approach will augment the odds of detecting a signal by broadening our understanding of the evolutionary and systemic components in the search for extraterrestrial intelligence (ETI), provide more targets for radio and optical SETI, and identify new ways of decoding and coding messages using universal markers. Key Words: SETI-Astrobiology-Coevolution of Earth and life-Planetary habitability and biosignatures. Astrobiology 16, 661-676.
\end{abstract}

\section{Introduction}

$\mathbf{T}$ HE KePLer MISSION and ground-based observatories have revealed thousands of exoplanets in small sectors of our galaxy alone, thus providing powerful evidence that our solar system is not an exception but simply one out of countless others in the Universe (e.g., Borucki et al., 2010; Kopparapu et al., 2013; Seager, 2013; Batalha, 2014; Gaidos et al., 2014; Hatzes, 2014; Quintana et al., 2014; Macintosh et al., 2015). What remains unanswered, however, is whether life-simple or complex-exists beyond Earth.

For nearly 20 years, astrobiology has brought a multidisciplinary vision to this quest through three fundamental science questions: (1) How does life begin and evolve? (2) Does life exist elsewhere in the Universe? (3) What is the future of life on Earth and beyond? In the 2008 version of its roadmap (Des Marais et al., 2008), astrobiology proposed a global approach to these questions through a broad research program with the goals to understand the formation and evolution of habitable planets, to explore and characterize the evolution of planetary environments favorable to life's

${ }^{1}$ SETI in the context of this article means SETI research (as it is performed around the world) and should not be confused with the SETI Institute. In the cases where mention is made of the SETI Institute, this is clearly spelled out. development in the Solar System and beyond, and to find methods to detect the signatures of life on early Earth and other worlds.

Thirty-five years before the creation of astrobiology, a very similar intellectual approach had been articulated by Frank Drake in the "Drake equation" (Section 2) but with a different intent. The Drake equation provided a probabilistic model to estimate the number of actively communicating extraterrestrial civilizations in the Milky Way, and was formulated around a technological imperative (radio astronomy) and a philosophical question: Are we alone?

Decades of perspective on both astrobiology and the Search for Extraterrestrial Intelligence (SETI) show how the former has blossomed into a dynamic and self-regenerating field that continues to create new research areas with time, whereas funding struggles (Garber, 1999) have left the latter starved of young researchers and in search of both a longterm vision and a development program. A more foundational reason may be that, from the outset, SETI is an all-or-nothing venture where finding a signal would be a world-changing discovery, while astrobiology is associated with related fields of inquiry in which incremental progress is always being made.

Yet in the same way astrobiology approaches the understanding of life in the Universe, SETI carries in its quest fundamental and unique questions that are central to the 
understanding of who, what, and where intelligent life is, and how to find it. While Are we alone? is their philosophical and popular expression (Zuckerman and Tarter, 1979; Tarter and Chyba, 1999), their scientific formulation can be expressed in these questions: How abundant and diverse is intelligent life in the Universe? How does intelligent life communicate? How can we detect it? If understanding life is the thread woven through the astrobiology roadmap, understanding how intelligent life interacts with its environment and communicates information is central to SETI.

In the following sections, we examine how scientific and technological advances are now allowing us to explore these questions in ways that were not previously possible, and to build the foundation of a long-term, multidisciplinary, and integrated vision for SETI. Here, we offer a perspective on how we could scientifically augment the odds of finding extraterrestrial life (ET) and an invitation to change our mind-set in doing so.

\section{Historical Pathway to ET}

While the scientific foundation for a living universe was established in the $16^{\text {th }}$ century with the Copernican revolution, the nature of advanced civilizations remained the domain of philosophers and fiction writers for a few more centuries (e.g., Descartes, 1644; de Fontenelle, 1686; Voltaire, 1752/2002; Kant, 1755/2009; Swendenborg, 1758; Flammarion, 1872_reviews by Dick, 1984; Crowe, 2011). The latter populated the Universe and our psyche with beings and worlds that were no more than idyllic or nightmarish versions of ourselves, our society, and our biosphere. By the end of the $19^{\text {th }}$ century, the advent of new technologies opened a different epistemological chapter for SETI. At that point, the quest for alien civilizations started to transition from a justifiable belief to a technology-based endeavor. Electricity and radio were first proposed as means to communicate with our close neighbor (Seifer, 1996) after Schiaparelli described channels_canali, in Italian-on Mars that later, Lowell (1906) erroneously claimed were artificial canals constructed by Martians. Over a half-century later, Cocconi and Morrison (1959) became the first to point out the possibility of searching for aliens in the microwave spectrum. Their expectation was that extraterrestrial signals could be pulse-modulated (e.g., Shostak, 2011a), and they proposed to examine solar-mass and red dwarf stars within 15-50 lightyears of Earth.

The conclusion of their article in Nature contained key comments that still resonate today. These comments include the realization that technology was finally available to test scientific hypotheses consistent with observational and theoretical astronomy, an understanding of the transformative philosophical and practical implications of a potential contact with an alien civilization (e.g., Almár and Tarter, 2011; Eliott, 2011a), and a concern that their work would be consigned to science fiction by many.

Independently from Cocconi and Morrison, astronomer Frank Drake had been formulating similar plans to conduct an actual search, and within a year of the publication, Project Ozma was launched at Green Bank (Schuch, 2011). Modern SETI was born and set out to search for ET's presence through narrowband radio astronomy. Broadband optical astronomy became an additional search tool in 1998 with OSETI (Ansbro, 2001), although the idea was previously suggested by physics Nobel laureate Charles Townes, whose research led to the maser and the laser (e.g., Schwartz and Townes, 1961; Wright et al., 2014).

Since 1961, SETI's intellectual framework has been centered on a probabilistic argument, the now-famous Drake equation:

$$
N=R_{*} \cdot f_{\mathrm{p}} \cdot n_{\mathrm{e}} \cdot f_{\mathrm{l}} \cdot f_{\mathrm{i}} \cdot f_{\mathrm{c}} \cdot L
$$

where $N$ is the number of civilizations in the Milky Way whose electromagnetic emissions are detectable; $R_{*}$ is the average rate of star formation in our galaxy; $f_{\mathrm{p}}$ is the fraction of those stars with planetary systems; $n_{\mathrm{e}}$ is the number of planets, per solar system, with an environment suitable for life; $f_{1}$ is the fraction of these planets that actually develop life; $f_{\mathrm{i}}$ is the fraction of life-bearing planets that develop intelligent life; $f_{\mathrm{c}}$ is the fraction of civilizations that develop a technology that releases detectable signs of their existence into space; and $L$ is the length of time such civilizations release detectable signals into space. For reviews and discussions about the Drake equation, see, for example, the works of Shostak (1998), Vakoch and Harrison (2011), Maccone (2012), Vakoch (2014), Vakoch and Dowd (2015), and Darling and Schulze-Makuch (2016).

Both lauded and criticized from the day it was formulated, the Drake equation clearly represented a watershed moment in science that gave humanity a glyph with which to spell alien life for the first time. Included as a forethought to the 1961 Green Bank Search for Extraterrestrial Intelligence meeting agenda (Drake, 2011), the so-called equation proved to be anything but. Consisting of a combination of quantitative and speculative factors that were solely meant to engage a discussion within the scientific community on the potential number of extraterrestrial civilizations willing and able to communicate in our galaxy, it quickly became SETI's signature. In hindsight, it was much more.

\section{A Roadmap}

The term equation is misleading in this context. Nor does the label probabilistic argument fully capture what the formulation truly represents, and the succession of some of its variables could be criticized. For instance, between $f_{\mathrm{i}}$ and $f_{\mathrm{c}}$ the notion of intelligence abruptly gives way to that of civilization, when they are, in fact, distinct notions. On our planet, one has not been demonstrated to necessarily lead to the other. Earth has developed a number of intelligent species, some of which use tools, while others are organized in hierarchical societies. Ultimately, the term technological civilization is what differentiates humankind, that is, an advanced stage of social development and organization, where scientific knowledge is applied for practical purposes on an industrial scale. In our case, this technology allows our species to scientifically explore and characterize our planet, the Solar System, and the Universe - and, on Earth, this is unique to the human species.

Nevertheless, the Drake equation was essentially the first roadmap and the first holistic vision of the search for life in the Universe (Tarter, 2007) — an approach that would become the trademark of the nascent field of astrobiology 35 years later (Des Marais et al., 2008). Even at this seminal 
time, it captured in one expression the notions of extrasolar planetary systems, habitable zones, habitable planets and environments, the transition from chemistry to biology, life on and beyond Earth, and the evolution of intelligence, civilization, and technology.

In the epistemological sense, the Drake equation provided a reductionist approach by scientifically breaking down the question of technologically advanced civilizations into simpler and smaller parts (the variables). Ultimately, it overlooked its own revolutionary vision as SETI focused on the application of one technology to search for Sun-like stars and red dwarfs (e.g., Tarter, 1979, 2004; Billingham et al., 1980; Gulkis et al., 1981; Heath et al., 1998; Turnbull and Tarter, 2003; Tarter et al., 2007). This rationale remains largely unchanged today despite advances in exploration and science that are relevant to the factors of the equation. As it stands, SETI does not search for all life or for all intelligent life. It focuses exclusively on technologically advanced life.

At the time of its formulation, the Drake equation was simply meant to be a tool to gauge how many technologically advanced civilizations might be "out there." The focus was clearly on the end-number $(N)$ to evaluate whether radio astronomy and astrophysics could provide successful strategies to intercept ET's signal across interstellar distances (Tarter, 2001a). The focus was not on trying to understand or quantify the processes taking place within each of the factors that lead to the next. Drake himself stated that his equation was not aimed at searching for primordial or primitive life-forms (Drake, 2011). Rather, it was aimed at the radio search. This explains SETI's path from the moment the Tatel Telescope at Green Bank was turned toward Tau Ceti and Epsilon Eridani for the first time. This might also explain why the radio receivers have remained silent since (see Sections 4 and 5).

Over the years, and with the exception of a clear connection to exoplanet detection (Tarter, 2001a, 2001b; Harp et al., 2015) and other astronomical fields, SETI around the world has evolved, for most, independently from core disciplines that rightly should be considered central to its understanding of what alien life could be and, therefore, to an understanding of how to optimize a detection strategy. While individual efforts exist, they have yet to be integrated into one vision and effectively support each other and existing projects (e.g., Fig. 1). It could be argued, but with only partial justification, that the reasons for SETI's all-ornothing venture are grounded in historical circumstances. Very little data were available to quantify the factors of the Drake equation at the time it was formulated, and most knowledge about the Solar System and the Universe was still primarily coming from ground-based astronomy and astrophysics. President Kennedy was a year away from declaring the race to the Moon, and orbiters were sent to Mars and Venus for the first time. It was a time of discoveries to come, in which the young science of radio astronomy was viewed as a unique tool to help humanity make what would be a world-altering discovery.

\section{The Evolution of $\boldsymbol{N}$ in the Past Decades}

By contrast to this time, large databases are now available in many relevant fields - and not necessarily only in as- tronomy-but their integration to SETI's approach has been slow in coming. Yet the means are currently in place to substantially increase the odds of finding ET through the development of an expanded search strategy that reflects this new knowledge.

\subsection{Searching for ourselves}

Part of this expanded vision starts by acknowledging that so far, in our quest to find ET, we have only been searching for other versions of ourselves, making the odds of success possibly more daunting than already dictated by nature. While the first variables of the equation are astronomical, observable, measurable, and most likely universal, as we move to the right, they become local, and the transition from life to intelligence to civilization and technology does not even characterize the average life-form on Earth. It characterizes us humans, a statistical outlier on our own planet.

Searching for other versions of ourselves is not unique to SETI. This is equally true of astrobiology, and it makes perfect sense as a starting point. After all, this is the only model of life we know, and a model that has been proven on our planet. Such an exploration strategy is further vindicated by the fact that the basic elements of life as we know it (CHONPS) are common in the Universe (Bada et al., 1994; Dyson, 1999; Seckbach et al., 2004; Brack, 2005; Mandell, 2008; Gibb, 2013), and habitable environments for life as we know them seem plentiful. Moreover, the Kepler mission and ground-based data suggest that there could be as many as 40 billion Earth-like planets in our galaxy alone (Petigura et al., 2013), $25 \%$ of them around Sun-like stars. Other models suggest that there could be up to 700 trillion planets in the Universe, but the vast majority would be far older than Earth (Zackrisson et al., 2016). Using a Biological Complexity Index, Irwin et al. (2014) proposed the existence of $\sim 100$ million planets in the Milky Way where complex life could have evolved. However, as they note, complex life does not necessarily mean technologically advanced life.

Although encouraging, large numbers do not tell the whole story. Zackrisson's study suggests that these worlds are likely to be very different from our planet, while other studies take the extreme position that Earth could be unique (Ward and Brownlee, 2003). In addition to the possible issues of age and composition, fundamental questions have now emerged from space, planetary, and earth sciences that directly bear on the question of planetary habitability as we know it, life beyond Earth, and its potential evolutionary tracks. These questions include the following:

Is there a generational aspect to the rise of life in the Universe depending on elements delivered by dying stars (e.g., Cowan and Sneden, 2006)? Did the configuration of the Solar System play a role in the successful development and survival of life on Earth (e.g., star, planets, their composition, size, and position)? In particular, did planets the size of Jupiter and Saturn protect life on Earth by attracting most cosmic debris (Horner et al., 2013) or, alternatively, foster the development of life on Earth by helping the delivery of volatile materials from the outer Solar System needed for life to form (Grazier, 2016)? Did our moon play a critical role in the rise of biology by stabilizing Earth's climate (Laskar et al., 1993; Lissauer et al., 2011)? How did Earth transition from prebiotic chemistry to life (Lazcano 


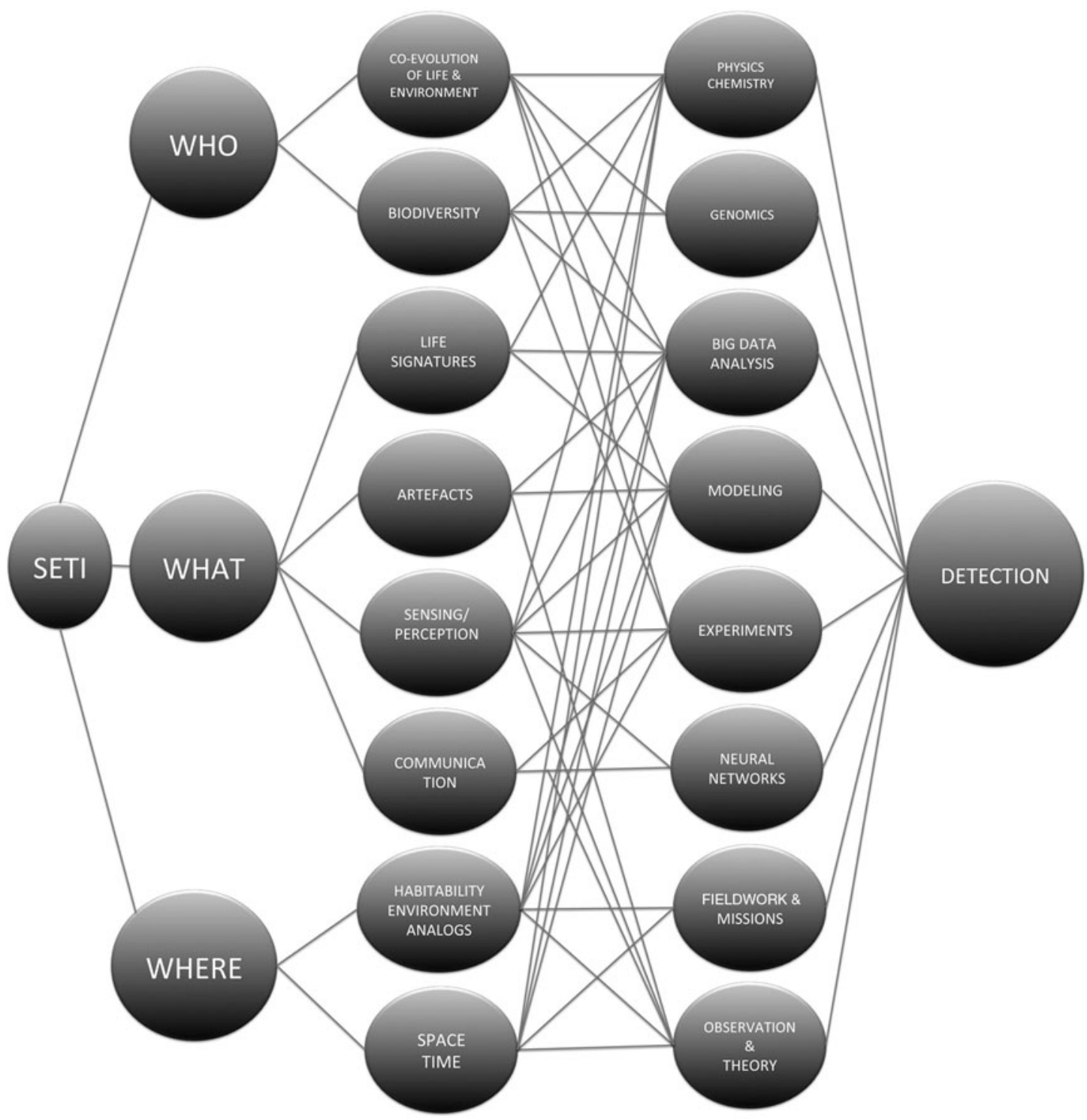

FIG. 1. Example of an integrated path to ET detection showing basic science questions (e.g., who and what are we searching for, and where?), key multidisciplinary science concepts, and relevant exploration methods (how do we investigate?). The combination of questions and approaches results in a directed and scientifically rich vision to ET detection, whose output is a reasoned exploration target. Since 1960, SETI's exploration strategy has prioritized the detection method (radio and optical searches) without addressing the fundamental nature of who and what ET could be, which has fundamental implications for where and how to search for it. The science concepts and methods shown here are discussed in the text.

and Miller, 1996; Pascal et al., 2006; Fitz et al., 2007; McCollom, 2013; Ruiz-Mirazo et al., 2013; Imari Walker, 2014; Walker, 2014)? Does environmental stability foster or inhibit life's inception, survival, and evolution; or does it take a mix of stability and chaos to stimulate biochemistry and biodiversity (Rosenfeld, 2011)? Do plate tectonics and oceans play a critical role in prebiotic and biotic processes (Spohn, 1991; Heller et al., 2011; Stern, 2015) —and are they critical individually or collectively (Kasting, 1993; Martin et al., 2008; Golding and Glikson, 2011; Lyons et al., 2014)? The most basic of all these questions remains today without an answer: What is life?

These are just a few examples, but the answer to each question has the power to deeply shape the vision of what the potential for an "Earth-like", planet to harbor life really means, and will affect the probability for such a planet to develop advanced and technological life.

\subsection{Evolutionary and systemic approach to $\mathrm{N}$}

Progressing to the right of the Drake equation, astronomy and biology are linked through two concepts: habitable zone and environmental habitability (e.g., Cockell et al., 2016). Both notions are mutable in space and time, the latter even more so than the former; and on Earth, climate change, extreme terrestrial environments, and genomics demonstrate how fast environmental changes can happen and how much the evolution of life and environment are intertwined (Golding and Glikson, 2011). While the initial astronomical and physicochemical conditions may drive the type of 
prebiotic chemistry possible on a planet, life, as demonstrated on Earth, becomes a primary player in shaping its environment as soon as it takes hold (e.g., atmosphere, landscape, mineralogy). This is the fundamental concept of coevolution of life and environment (e.g., Watson, 1999; Kooijman, 2004; Dietrich et al., 2006; Knoll, 2009; Grenfell et al., 2010; Kolb, 2014). This coevolution will dictate the uniqueness of each planetary experiment (Irwin and Schulze-Makuch, 2001; Schulze-Makuch et al., 2013) and will do so not only when (or if) life reaches the stage of technological advancement. It will start from the very first moment, as it did on Earth.

Earth's biosphere, and all events that led to us, and the way we perceive the environment and the Universe are the result of over 4 billion years of reciprocal influences between environmental and biological processes (Fabbro et al., 2015; Houri-Ze'evi et al., 2016). They are the result of evolutionary bottlenecks induced by geological, climatic, and cosmic events (Lynch and Lande, 1993; Bürger and Lynch, 1995; Bijlsma et al., 2013). An asteroid impacting instead of missing Earth would completely alter the course of evolution at any time.

Ultimately, biological evolution on Earth has been partially dictated by somewhat predictable cycles and events (astronomical, climate) but drastically more so by stochastic events, both geological and biological in nature (Fig. 2) (Pigliucci and Müller, 2010), by when and where they occurred and at what stage of evolution they happened (Gould, 1998; Raup, 1999; Schulze-Makuch and Irwin, 2001; Schulze-Makuch et al., 2013; Keller and Kerr, 2014; Lehman and Miikkulainen, 2015). It follows that, even considering panspermia and planetary exchange as primary seeding mechanisms (e.g., Arrhenius, 1907; Arrhenius and Mojzsis, 1996; Melosh, 1996; Burchell et al., 2004; Napier, 2004; Gladman et al., 2005; Owen, 2008; Worth et al., 2013), two planets with absolutely similar environments and receiving absolutely similar seeding material may have two very distinct biological destinies depending on when extinctions and evolutionary bottlenecks take place, and whether these events have transient or long-lasting effects

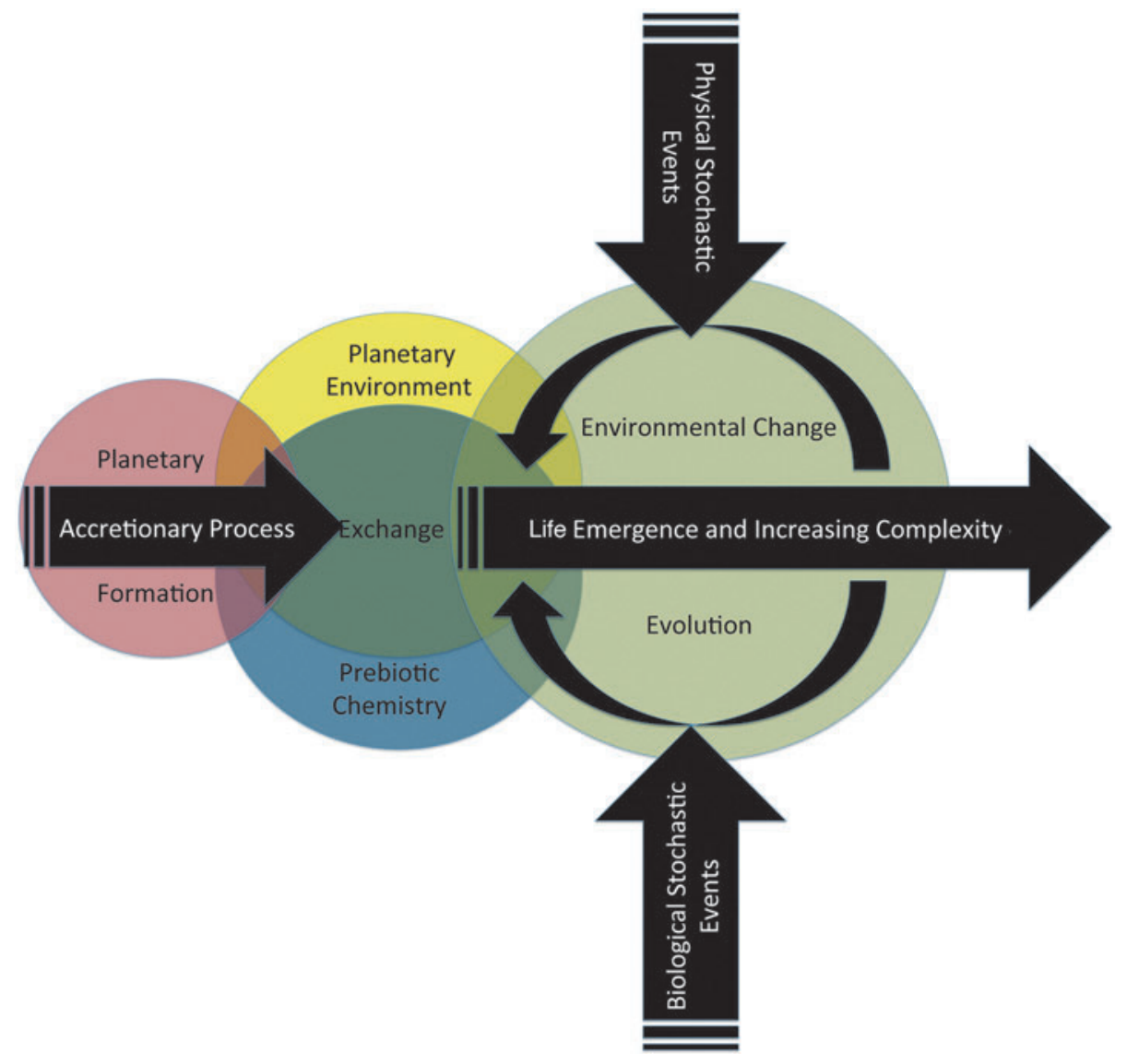

FIG. 2. Coevolution of life and environment. Environmental perception and neural systems of the species living on Earth are intimately linked to the coevolution of life and environment, a process that has been subjected to random probability events all along its continuum. This process started with accretion and the specific elements that contributed to planetary formation. As prebiotic chemistry transitioned to life, the period of heavy bombardment continued to spatially randomly deliver material to Earth, contributing equally to the destruction of a nascent life and the development of a new one. Since life took hold, stochastic events have continued in the physical world as environmental and cosmic catastrophes, and in the biological world as adaptive evolution and epigenetic changes. While the notions of habitable zone and environmental habitability are critical in trying to predict whether a planet could host life as we know it, the very random nature of the coevolution of life and environment renders each planet and the life it may bear a unique experiment, which questions the anthropocentric principle underlying the Drake equation. 
on the environment. Stochastic events will give each planet a unique fingerprint. If life arises through abiogenesis of simple organic compounds (Des Marais, 1999; Popa, 2004; Shapiro, 2006; Pross, 2012; Pross and Pascal, 2013), this fingerprinting will be even more uniquely connected to its planet of origin, and cosmic chances are that both exogenic and endogenic processes are acting together in proportions specific to each planetary system.

Moreover, if Earth is any indication of universal planetary laws of evolution, nature produces simple systems in overwhelming numbers compared to complex systems (Mandelbrot, 1982). Statistically, microbial life still dominates today, and for $70 \%$ of Earth's history was the only form of life on our planet (Margulis, 1996; Nealson and Conrad, 1999; Wolf et al., 2002). Taking inventory, global species richness estimates suggest that up to 10 million species of eukaryotes and up to 100 million to a billion prokaryote species exist today on Earth (Colwell and Coddington, 1994; Gaston, 2000; Colwell, 2009; Mora et al., 2011; McGinley, 2014), while the most recent studies model up to one trillion species (Locey and Lennon, 2016). In this inventory of all terrestrial life, through competition, only humans have reached technology. However, this statistical snapshot alone does not provide an accurate estimate of a realistic ratio, as it does not reflect the (unknown) total number of species produced by our planet over time. Those include evolutionary dead ends and species that went extinct as a result of climatic or catastrophic events but still played their part in shaping the global biosphere from which we, the human species, emerged. It does not account for the shadow biosphere hypothesis, either (Cleland and Copley, 2005; Davies et al., 2009).

A systemic approach to $N$ shows that it took 4 billion years of symbioses, competition, biodiversification, and the development of all species that did-and did not-survive for us to be here. Some key evolutionary leaps have yet to be completely understood. Only very recently, the common ancestral gene that may have enabled the evolution of complex life over a billion years ago was identified. Without this gene, life on Earth might not have evolved beyond the stage of slime (Lai et al., 2016). We also have yet to understand what led one species of apes to separate itself from the others just a few million years ago (Arsuaga, 2010). Understanding these transformative processes may bear considerable weight on $N$. It is certainly not necessary to wait for all the answers to start searching for ET, but we must acknowledge that these questions exist, and develop search strategies adapted to the complexity they underscore in order to augment the chances of success.

We are, indeed, the product of local astronomical and planetary factors. However, it would be unreasonable to suggest that similar evolutionary convergence never happened with seemingly so many planets already discovered in the small spatiotemporal window of the Kepler telescope. Somewhere out there, based solely on numbers and probabilities, life may have evolved to bear some resemblance to us - if only fortuitously. It might interact with its planetary environment as we do, and evolve to produce biological forms with logical minds presenting similarities to us who may be willing to communicate in ways we can understand. However, the numbers are unlikely to be in the billions or even the millions in our galaxy. There may be just a handful scattered across vast distances and time.
Taking life's evolution on Earth as a guide, there is likely a universal probabilistic law of evolutionary convergence that is inversely proportional to life's complexity; that is, the simpler life is, the greater chances are that similar life-forms will be abundant throughout the Universe. The more complex life is, the more rare convergence is likely to be. Complexity in life-forms is an integration of temporal evolution and probabilistic events. The longer life is around, the greater chance it has to adapt through regular cycles and, at any given time, to mutate through stochastic events. Looking back at ourselves, it took $70 \%$ of Earth's time in the habitable zone and an incredible amount of "chance and necessity" (Monod, 1972) for one species in a complex tree of life to reach civilization and technology.

The longer evolution takes, the greater the chances are that species will be wiped out and ecosystems profoundly transformed (e.g., Alvarez and Asaro, 1990), but with the rise of technology, some of the endogenic and exogenic risks to life can also be offset (e.g., asteroid monitoring, Yeomans, 2013). Conversely, human evolution shows that technology brings its own sets of risks: the natural dynamics are upset (Holocene extinction: Barnosky et al., 2011), the environment modified (Anthropocene: Grinspoon, 2012; Waters et al., 2016), and the terms of the coevolution of life and environment that led to the rise of the dominant species deeply altered.

At this point in time, humans have generated an environmental disequilibrium that reverberates across the biosphere globally and endangers the conditions of planetary habitability that were favorable to its emergence. The notion of self-engineered destruction is certainly present in the last factor of the Drake equation. $L$ reflects on how long a technological civilization might be willing and able to communicate. More than duration, this factor focuses on the odds of detecting a signal; that is, the longer an alien civilization broadcasts its presence, the more chances we have to detect it. Assuming the anthropocentric view of a technological civilization presenting similarities with ours, willingness to communicate may depend on a host of reasons (e.g., political, scientific, technological, philosophical, religious, and social). How long such a civilization would continue to communicate is a more complex issue. Duration can relate to a civilization's ability to avoid self-inflicted-or other-destruction, scientific advances, and interest. It could also relate to a cosmologic perspective we have not yet reached, including a sense of place and responsibility as a member of a universal community (e.g., the Fermi paradox).

As expressed, $L$ examines for how long such a civilization would broadcast a signal in ways we can detect, which are primarily focused on radio and optical astronomy. We could assume, however, that a technological civilization may communicate or broadcast in ways so advanced compared to ours that we simply cannot imagine what they are and are thus unable to detect its signals. Or this civilization may have long since disappeared. These scenarios are implicitly present in the current definition of $L$. However, there is also an evolutionary dimension to this factor that transcends the existing formulation.

\section{Expanding the $\mathbf{N}$ Horizon}

The evolutionary pathways that lead to complex life on Earth strongly suggest that advanced life as we know it may 
be rare in the Universe and unlikely to be in a state of advancement that is temporally synchronous with us. However, that does not mean that other types of advanced intelligences are as rare. Limiting our search to something we know and can de facto comprehend is, probabilistically, a constraining proposition, one that leaves no room for an epistemological and scientific foundation to explore alternate hypotheses. To find ET, we must expand our minds beyond a deeply rooted Earth-centric perspective and reevaluate concepts that are taken for granted.

\subsection{Becoming aliens}

Rather than constraining the search, SETI efforts must involve the most expansive exploration tool kit possible. If we unbind our minds, it should not matter whether ET looks or thinks like us, has a logic that makes any sense to us, or uses familiar technology for interstellar communication. ET is likely to be very different from us and completely alien to our evolutionary processes and thought processes, which may be deeply connected (see Section 5.2.3). Ultimately, to find aliens, we must become the aliens and understand the many ways they could manifest themselves in their environment and communicate their presence.

Such an intellectual framework not only moves the Drake equation forward toward the existence of drastically different probabilistic civilizations, it also brings us to consider alternate evolutionary pathways, including life as we do not know it and do not yet understand. Further, such a framework allows us to look at evolutionary pathways in our own biosphere and question the emergence of complex, intelligent life with a different set of eyes. For that to happen, we must conceptualize something we do not know, which can be approached in a number of different ways. One is by trying to access unknown concepts and archetypes that are literally alien to us (i.e., not part of our own evolutionary heritage) through imagination and discourse. This is what science fiction attempts to do in its depictions of alien worlds and civilizations. Not surprisingly, this process results in more or less elaborate versions of ourselves, since these representations are generated by neural systems wired to our own planetary environment. To conceptualize a different type of life, we have to step out of our brains.

\subsection{Universal heritage}

A path to finding life we do not know requires us to identify a common universal heritage, one that includes signatures and signals that can be recognized across different evolutionary tracks and across space and time. Research in this direction was initiated by the Communication with Extraterrestrial Intelligence (CETI) and the Arecibo message (Sagan, 1975; Vakoch, 2011a). Advances in astrobiology, life science, and cognitive science are bringing new perspectives and depth to that concept. Some of them are already being explored, while others belong to disciplines that have yet to be involved with SETI and represent a currently untapped potential.

5.2.1. Distant biosignatures. In searching for life in the Solar System and on exoplanets, astrobiology is using an approach based on the concept of "universal heritage" but more narrowly focused on life as we know it (Seckbach, 2006). For instance, water and carbon are driving search strategies; the formation, preservation potential, and detection methods of biosignatures that could be similar to Earth's are being investigated for the exploration of extinct and extant life on Mars, Europa, and exoplanets. In situ biosignatures are physicochemical, geological, morphological, and mineralogical in nature (Summons et al., 2011). Remotely detectable biosignatures include gases in planetary atmospheres (Pilcher, 2004; Segura et al., 2005; Domagal-Goldman et al., 2011; O'Malley-James et al., 2014; Seager, 2014; Krissansen-Totton et al., 2016). Given an environmental analogy, it is conceivable that alien biospheres presenting similarities with ours may have generated and left traces we could recognize. However, none of these signatures are convincingly unambiguous evidence of life's presence as both biological and abiotic processes alike can produce them (Schwieterman et al., 2016). Therefore, it might be difficult to use them as universal markers of life as we know it, let alone for life we do not know.

Astrobiology and Earth sciences show that the systemic disequilibrium generated by the presence of life could be a promising candidate as a universal marker of life (Schwartzman, 2004; Branscomb and Russell, 2013, Russell et al., 2013). Biological activity, from microorganisms to humans, utilizes and modifies its environment, producing traces (physical, chemical, isotopic) not otherwise found in nature in the absence of life. As long as we search for biology with a physicochemical support, such disequilibrium will be generated and measurable across species and planets - although we will have to start by learning how to untangle it from the planetary background. The argument can also be made that some technological civilizations, or civilizations beyond technology, may be so advanced that they have returned to equilibrium and generate living conditions that do not betray their physical presence anymore-or they purposely hide their presence (Kipping and Teachey, 2016). In such instances, they will remain stealth to this search method. Planetary biosignatures reflecting the presence of a biosphere will still be visible, but traces of advanced beings on that planet may no longer be detectable.

While this marker falls short of helping the detection of environmentally stealthy civilizations, it can help find those that have not yet reached that stage. It also offers a universal vision to life detection that goes beyond life we know, thereby vastly expanding the statistical planetary pool that can be probed today. Further, and critical for SETI, this approach does not depend on the willingness of aliens to communicate. The coevolution of life and environment creates a physicochemical overprint that, regardless of the stage of life's development, will betray its presence. Learning the range of signatures produced by such disequilibrium from local to global scale should, therefore, become a priority in the development of techniques to search for life of all types, sizes, and advancement stages. Even though each candidate signature might not be produced by an advanced civilization, this method will identify more potential targets for SETI. The current limitation is both the spectral resolution of instruments and the distance of possible targets, but this method should become a critical investigation strategy to survey our galactic neighborhood (Seager and Deming, 2010; Deroo et al., 2012; Beichman et al., 2014; Burrows, 2014; Seager, 2014; Crossfield, 2015). A search for systemic disequilibria at a planetary scale is currently one of the most promising methods for detecting 
life beyond Earth. Taken alone, it might not be enough to inform us of the stages of life's development, but in the coming years, analog missions to our own atmosphere might teach us how to identify technosignatures.

5.2.2. Communication. Ultimately, the evidence SETI is looking for is an unambiguous signal from ET (e.g., Tarter, 1983; Shostak, 1998). To intercept alien signals, SETI has traditionally relied on radio astronomy for passive listening, and more recently on optical methods, and while the idea of active SETI-also known as Messaging Extraterrestrial Intelligence (METI) - remains a subject of controversy (Atri et al., 2011; Vakoch, 2011b; Vakoch and Matessa, 2011; Neal, 2014), communication and contact are very much at the core of the SETI concept. But what are we searching for and listening to?

In theory, SETI is searching for coded messages sent through controlled laser emissions or radio signals containing patterns that cannot be readily explained by known natural phenomena, (e.g., Tarter, 2001b; Vakoch, 2011a; Forgan, 2014; Wheeler, 2014; Heller and Pudritz, 2016). Some research areas focus on the type of messages that should be broadcast if/when METI becomes active and on the content of these messages (Eliott, 2011b, 2011c; Ollongren and Vakoch, 2011; Vakoch, 2011b, 2011c; Musso, 2012). Since physical and mathematical principles are universal, they can be considered part of a universal heritage and a good starting point for composing messages (Ilhan and Linscott, 2011; Azua-Bustos and Vega-Martinez, 2013). Symbols, geometry, visual imageries, music, and sounds have been used in the past as part of the Pioneer and Voyager messages (Rose and Wright, 2004; Shostak, 2011b). They continue to be investigated with the understanding that some might not be universal but biased toward senses humans have that ET might not have developed-or developed differently.

Since the ultimate goal of SETI is to identify alien signals, it should follow that understanding the origin, structures, and various forms and supports of communication (natural and artificial) is a fundamental step in the development and success of the search (Witzany, 2015). Research in marine and land species has already revealed common patterns that may be clues to laws of communication in the languages of some of Earth's intelligent species (Doyle et al., 2011). This research area shows important promises, and these patterns might help us identify and decrypt alien messages coming our way one day. However, an important question-whose answer may lie in genomics and neuroscience-is whether these shared patterns are Earth-specific and the result of common environmental context and evolution, or actually universal in nature.

Further, perception and communication are severely constrained by sensory range (e.g., wavelengths, amplitude, reach) and by words in humans. The complexity and subtlety of a thought or a feeling can often be lost when it reaches verbal formulation even for members of the same cultural and linguistic groups. This limitation will be more acute between interplanetary species that evolved on planets with potentially different physicochemical conditions, environmental contexts, and mixings.

5.2.3. Environmental perception-alien neural systems. Most advanced alien species will likely have developed forms of communication completely unrecognizable to us. It can be hypothesized, however, that life anywhere must gather information about its environment for purposes of development, adaptation, survival, and evolution (Geary, 2005; Roth, 2013; Krubitzer, 2014; Schulkin, 2014). For terrestrial species, information is collected through senses, including many shared across species, while others are species-specific (e.g., echolocation, electroreception, magnetoreception). The senses are transducers from the environment to the brain where information is being interpreted-or through homolog systems for species without a nervous system (Strausfeld and Hirth, 2015). Alien life may need equivalent systems to process and integrate internal and external information. This is how ET will perceive its environment (e.g., Nolfi and Parisi, 1996; Pfeifer and Gómez, 2006) and communicate information about it in the messages it encodes, and for us, this is the key to unlock how we will decode them.

In that respect, cognitive and mathematical sciences (e.g., neuroscience, computational modeling, machine learning, other) may be the disciplines that can deliver the most transformative advances to SETI at this point in its history. By understanding how perception, concepts, and archetypes are being formed and formulated (Perlovsky, 2006) and how information is being stored and processed through neural or homolog systems, SETI could start exploring the source point of communication, where the elemental components of perceiving, interacting with, and communicating our understanding of planetary environments, our place in the Universe, and the Universe itself are being generated.

The structure and content of any alien message will have a local overtone to these elemental components driven by where (space and environment) and when (time) a civilization appeared in the Universe. However, on the cosmic scale, physics, chemistry, and biology are constrained by universal parameters that link all life-as diverse as it may be. These parameters are our common heritage and the letters of our universal alphabet. Life sciences, and cognitive and mathematical sciences, provide a fundamental and mandatory path for SETI to understand this universal alphabet. They are already used in the investigation of possible universal languages (Vakoch, 2011a). However, in order to decode or encode messages from and to ET, a broader approach than the existing one is needed, one that considers the many possible combinations of evolutionary paths-not just those most familiar to us.

What SETI needs is a comprehensive integration of the concept of coevolution of life and environment in its search strategy, and the essential role it may play in the structure of ET's environmental sensing systems. That coevolution may fundamentally affect how aliens perceive their world, intellectualize it, and communicate it. As our knowledge of exoplanetary environments broadens, we are becoming better prepared to conceptualize the potential biochemistries and types of life these environments could support. We must use this background to develop probabilistic simulations of alien neural_-or homolog sensing —-systems. This will allow us to see the environment and the Universe as ET may perceive them, and not as we hope it does with our own set of senses. Without that basic understanding, SETI will essentially continue to search blind.

Such a research avenue can now find a robust scientific foundation in the advances made by evolutionary biology in innovative areas such as evolvability, evolutionary 
developmental biology, epigenomics, and system biologyregrouped under the term of extended synthesis (Pringle, 1951; Smith and Cribbs, 1994; Ziman, 2000; Wagner, 2007; Wagner et al., 2007; Pigliucci and Müller, 2010; Wagner and Draghi, 2010; Schrey et al., 2012; Jerison and Jerison, 2013; Valiant, 2013; Badyaev and Walsh, 2014; Watson and Szathmáry, 2015). This body of work strongly suggests that life, intelligence, and perception are shaped by, and designed to respond to, a planetary environment; this has powerful implications for SETI's anthropocentric principle and implications for its search strategy that cannot be ignored.

Today, vast amounts of data and computational capacity are at hand (Graham et al., 2005; Heinecke et al., 2015), allowing us to start on the path of modeling probabilistic evolutionary tracks using known exoplanetary environments, Solar System exploration, the exploration of extreme environments, and the computational tools of life, mathematical, and cognitive sciences and neuroscience (e.g., Wolpaw and Winter Wolpaw, 2012; Rao et al., 2014). Results may lead us to first identify, then design, novel types of communication methods (e.g., message content and support), instruments, and technologies to search for ET.

Further, while evolutionary biology holds critical clues for our understanding of advanced intelligent life in the Universe, the implications of its applications to modeling alien life are much broader than SETI alone. As new theories mature through validation and falsification, they will help us interrogate more deeply the evolutionary continuum of intelligence on Earth from simple to complex life, a process highly relevant to astrobiology.

Ultimately, though, while understanding evolution, perception, and communication is absolutely essential to SETI, it will not be impactful if this knowledge remains compartmentalized. Data generated by each of these disciplines must be fused together and transformed into reasoned search strategies and experimental protocols. They must result in the identification and articulation of science questions and hypotheses that open pathways forward to theories and quantifiable metrics and milestones (see also Fig. 1).

\section{An Integrated Vision Moving Forward}

So far, a number of perspectives have been offered to explain why radio receivers have remained silent for the past 50 years, including the Fermi paradox and the Zoo hypothesis (Ball, 1973). More recently, two new scenarios have been added: ET is dead, based on the argument for a Gaian bottleneck (Chopra and Lineweaver, 2016), and the opposite view, that we are the oldest civilization in the Galaxy (Sasselov, 2012). It may be that the answer is much simpler and humbling. The fact is that we embarked on this journey only a few decades ago and have applied to it very limited tools and detection strategies. Telescopes have become bigger, arrays larger, resolution better, but the technology and exploration strategies behind SETI have remained basically unchanged. In reality, we have yet to articulate core questions to place SETI in its proper, systemic, and evolutionary context.

In its current scientific and technological form, SETI can be likened to sampling the cosmic ocean with a plankton net, where the size of the antennas and their number are analogous to the mesh size. Refining the mesh size by adding antennas or making them bigger will not make the tool more effective compared to the breadth of exploration at hand. Further, this tool is only focused toward testing one very specific, largely anthropocentric, hypothesis about extraterrestrial intelligence (ETI), when data increasingly suggest that there are probably as many distinct life-forms and intelligences as habitable planetary environments in the Universe. While the historical view of ETI is a valid hypothesis that must be tested, it is one among many, and we should proceed with it acknowledging that the chances of a rendezvous somewhere in time, and space, with that particular alien are remote.

When the odds are small and the object of a search unknown, the rule of thumb is to cast as large a net as possible to increase the chances of success rather than the opposite. In this case, a larger net is not solely a larger set of antennas or more abundant resources focused on one technology or one experiment (Billings, 2015). For SETI, it is critical to fully embrace the multidisciplinary approach that was scripted 50 years ago in the Drake equation and create a well-stocked and diverse tool kit to explore a vision that serves as an umbrella under which many disciplines will synergistically contribute advances to expand the search for alien life beyond the anthropocentric principle.

Some bridges are already in place and being investigated, such as the clear connections between the search for ET and exoplanet exploration (e.g., Tarter, 2007). SETI uses the Kepler catalog and ground-based telescope databases to target high-priority candidates. Prioritization criteria are currently limited to parameters for life as we know it (e.g., exoplanets in the habitable zone of their parent stars; unexplained signals from known exoplanetary systems, see Harp et al., 2015; Schuetz et al., 2015; Abeysekara et al., 2016). Those criteria will be refined over time as universal markers are being identified and as we learn more about possible exotic biochemistries (e.g., McKay, 2004; Ward and Benner, 2007; Davila and McKay, 2014), but the connection is established and will only grow stronger.

In addition to this, new research pathways bear significant promises for a more universal SETI reach, such as ET and systemic planetary disequilibria, the coevolution of life and environment, and neural systems and their role in our perception of the Universe, our interaction with it and how we communicate about it - to name but a few. Figure 3 summarizes a path to achieving this integrated and synergistic vision. It shows a notional network between disciplines with bridges and research avenues connecting together space, planetary, life, geosciences, astrobiology, cognitive, and mathematical sciences. Others will be added as knowledge progresses. It is a dynamical vision forward, built on a connectivity network that represents an expanded version of the Drake equation, one that integrates all the historical factors now broken down into measurable terms.

This vision expands the search to life as we do not know it using universal markers and the disciplines, fields, and methods that will allow us to quantify them. Points of intersection are the knowledge that will be contributed to SETI by integrating these various disciplines. This contribution will be provided in five fundamental areas: (1) scientific rationale (theories, hypotheses); (2) experimental design (methods, protocols, and metrics); (3) communication (universal markers, signals, instruments, systems, technologies); (4) target identification; and (5) missions 


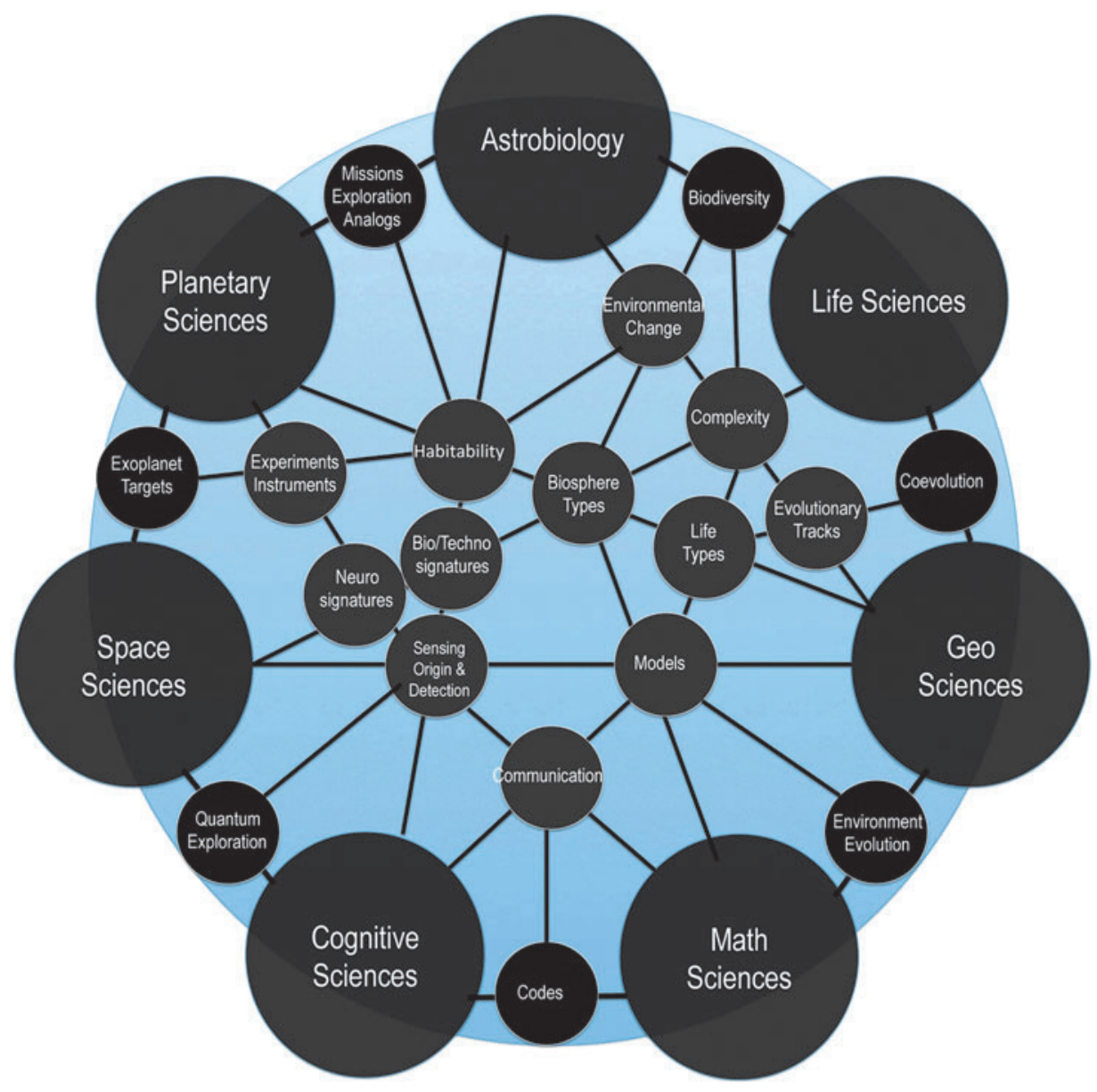

FIG. 3. Connectivity network between disciplines showing the bridges and research avenues that link together space, planetary, and life sciences, geosciences, astrobiology, and cognitive and mathematical sciences. This representation is an expanded version of the Drake equation. It integrates all the historical factors now broken down in measurable terms and expanded to include the search for life we do not know using universal markers, and the disciplines, fields, and methods that will allow us to quantify them.

that will allow SETI to implement its own vision. These sciences are already collecting vast data sets that can now be interrogated in ways we could not have imagined only a few years ago. Bioneural computing, communication theory, machine learning, neural coding, neural network and deep learning, data mining, and big data analysis are the building blocks of the connecting bridges that SETI must build as a prerequisite to a universal exploration of ET versus the current anthropocentric search.

\section{Conclusion}

Now is the time for SETI to develop a roadmap and longterm vision that includes the search for life as we do not know it. New tools are available that can enable this approach and help us decipher the evolutionary and probabilistic nature of advanced alien life. These tools are found in astronomy and astrophysics but also in the biological, geological, cognitive, mathematical, and computer sciences, among others. All of them must be deployed when thinking about who, what, and where ET could be, and how it might communicate.

To find ET, we will have to turn inward (evolution, perception, and communication) and outward (planetary environments) and challenge our anthropocentric assump- tions. Through consilience, we can build a systemic and connective vision of intelligent life in the Universe. To be achieved, this vision requires the exploitation of multidisciplinary synergies and an intellectual framework with a clear agenda and milestones. A similar intellectual and logistical structure was successfully established 20 years ago by NASA with the creation of the NASA Astrobiology Institute (NAI) for the understanding of life on Earth and beyond. However, past and present astrobiology roadmaps have not put substantial emphasis on alien intelligence, communication, or technology.

In the coming months, the SETI Institute will be initiating efforts in this direction, and will invite the United States and international research communities to contribute to the drafting of a new scientific roadmap for SETI. We will explore resources for the development of a virtual institute and an intellectual framework for multidisciplinary projects specifically focused on the advancement of knowledge on ETI. Complementary to, and different from, the astrobiology roadmap, this vision will bridge the NAI roadmap, augment it, and go beyond it.

Its primary goal will be to "Understand how intelligent life interacts with its environment and communicates." 
This will be explored through three main questions:

Question 1: How abundant and diverse is intelligent life in the Universe? With this question, SETI will synergistically use data from astrobiology, biological sciences, space and planetary science and exploration, and geoscience to quantitatively and qualitatively characterize the potential abundance and diversity of intelligent life in the Universe. Its spatiotemporal distribution will be approached using cosmological models of the physicochemical evolution of the Universe and inferred viable biochemistries.

Question 2: How does intelligent life communicate? Cognitive sciences, neuroscience, communication theory, mathematical sciences, bioneural computing, data mining, and big data analysis, among other disciplines, will explore communication in intelligent terrestrial species. They will use physicochemical and biochemical models of known exoplanetary environments to generate and map probabilistic neural and homolog systems, and infer the resulting range of viable alien sensing systems and the perception of the Universe they confer. Spatiotemporal distribution models will be inferred from Question 1-related investigations.

Question 3: How can we detect intelligent life? Exploration strategies, instruments, experimental protocols, technologies, and messaging (content and support) will be designed using the results, data, and databases of research conducted under Questions 1 and 2.

Ultimately, SETI's vision should no longer be constrained by whether ET has technology, resembles us, or thinks like us. The approach presented here will make these attributes less relevant, which will vastly expand the potential sampling pool and search methods, ultimately increasing the odds of detection.

Advanced, intelligent life beyond Earth is most likely plentiful, but we have not yet opened ourselves to the full potential of its diversity. With the vision presented here, we offer a unified and universal approach for the search for extraterrestrial life- one that is measurable and searches for ET at the crossroads of scientific and technological innovation, and imagination.

\section{Acknowledgments}

I am particularly grateful to those who, through conversations, constructive criticism, suggestions, comments, and reviews at various stages of development have helped me articulate this perspective. Special thanks to Bill Diamond, David Darling, Margaret Race, Mark Showalter, and Jill Tarter for their inputs. Also thank you to Maggie Turnbull and Laurance Doyle for sharing thoughts over the past few months.

\section{Author Disclosure Statement}

The author declares no conflict of interest.

\section{References}

Abeysekara, A.U., Archambault, S., Archer, A., Bendow, W., Bird, R., Buchovecky, M., Buckley, J.H., Byrum, K., Cardenzana, J.V., Cerruti, M., Chen, X., Christiansen, J.L., Ciupik, L., Cui, W., Dickinson, H.J., Eisch, J.D., Errando, M., Falcone, A., Furniss, A., Gillanders, G.H., Griffin, S., Grube, J., Gyuk, G., Hütten, M., Håkansson, N., Hanna, D., Holder,
J., Humensky, T.B., Johnson, C.A., Kaaret, P., Kar, P., Kelley-Hoskins, N., Kertzman, M., Kieda, D., Krause, M., Krennrich, F., Kumar, S., Lang, M.J., Lin, T.T.Y., Maier, G., McArthur, S., McCann, A., Meagher, K., Moriarty, P., Mukherjee, R., Nieto, D., O’Brien, S., O'Faoláin de Bhróithe, A., Ong, R.A., Otte, A.N., Park, N., Perkins, J.S., Petrashyk, A., Pohl, M., Popkow, A., Pueschel, E., Quinn, J., Ragan, K., Ratliff, G., Reynolds, P.T., Richards, G.T., Roache, E., Santander, M., Sembroski, G.H., Shahinyan, K., Staszak, D., Telezhinsky, I., Tucci, J.V., Tyler J., Vincent, S., Waleky, S.P., Weiner, O.M., Weinstein, A., Williams, D.A., and Zitzer, B. (2016) A search for brief optical flashes associated with the SETI target KIC 8462852. Astrophys $J$ 818, doi:10.3847/2041-8205/818/2/L33.

Almár, I. and Tarter, J. (2011) The discovery of ETI as a highconsequence, low-probability event. Acta Astronaut 68:358-361.

Alvarez, W. and Asaro, F. (1990) An extraterrestrial impact: accumulating evidence suggests an asteroid or comet caused the Cretaceous extinction. Sci Am 263:78-84.

Ansbro, E. (2001) New OSETI observatory to search for interstellar probes. Proc SPIE 4273, doi:10.1117/12.435370.

Arrhenius, G. and Mojzsis, S. (1996) Extraterrestrial life: life on Mars-then and now. Curr Biol 6:1213-1216.

Arrhenius, S. (1907) Panspermy: the transmission of life from star to star. Sci Am 96:196.

Arsuaga, J.L. (2010) Terrestrial apes and phylogenetic trees. Proc Natl Acad Sci USA 107:8910-8918.

Atri, D., DeMarines, J., and Haqq-Misra, J. (2011) A protocol for messaging to extraterrestrial intelligence. Space Policy 27:165-169.

Azua-Bustos, A. and Vega-Martinez, C. (2013) The potential for detecting "life as we don't know it" by fractal complexity analysis. International Journal of Astrobiology 12:314-320.

Bada, J.L., Bighamm, C., and Miller, S.L. (1994) Impact melting of frozen oceans on the early Earth-implications for the origin of life. Proc Natl Acad Sci USA 91:1248-1250.

Badyaev, A.V. and Walsh, J.B. (2014) Epigenetic processes and genetic architecture. In Quantitative Genetics in the Wild, edited by W.A. Charmantier, D. Garant, and L.E.B. Kruuk, Oxford University Press, Oxford, UK, pp 177-189.

Ball, J.A. (1973) The zoo hypothesis. Icarus 19:347-349.

Barnosky, A.D., Matzke, N., Tomiya, S., Wogan, G.O.U., Swartz, B., Quental, T.B., Marshall, C., McGuire, J.L., Lindsey, E.L., Maguire, K.C., Mersey, B., and Ferrer, E.A. (2011) Has the Earth's sixth mass extinction already arrived? Nature 471:51-57.

Batalha, N.M. (2014) Exploring exoplanet populations with NASA's Kepler mission. Proc Natl Acad Sci USA 111:12647-12654.

Beichman, C., Benneke, B., Knutson, H., Smith, R. Dressing, C., Latham, D., Deming, D., Lunine, J., Lagage, P.-O., Sozzetti, A., Sing, D., Kempton, E., Ricker, G., Bean, J., Kreiberg, L., Bouwman, J., Crossfield, I., Christiansen, J., Ciardi, D., Fortney, J., Albert, L., Doyon, R., Rieke, M., Rieke, G., Clampin, M., Greenhouse, M., Goudfrooij, P., Hines, D., Keyes, T., Lee, J., McCullough, P., Robberto, M., Stansberry, J., Valenti, J., Deroo, P.D., Mandell, A., Ressler, M.E., Shporer, A., Swain, M., Vasisht, G., Carey, S., Krick, J., Birkmann, S., Ferruit, P., Giardino, G., Greene, T., and Howell, S. (2014) Observations of transiting exoplanets with the James Webb Space Telescope (JWST). Publ Astron Soc Pac 126:1134-1173.

Bijlsma, R., Westerhof, M.D.D., Roekx, L.P., and Pen, I. (2013) Dynamics of genetic rescue in inbred Drosophila melanogaster populations. Conserv Genet 111:449-462. 
Billingham, J., Edelson, R., Gulkis, S., Olsen, E.T., Oliver, B.M., Tarter, J.C., and Seeger, C.L. (1980) A search strategy for SETI [paper \#IAA-80-57]. In $9^{\text {th }}$ International CETI Review Meeting of the International Academy of Astronautics, Tokyo, Japan.

Billings, L. (2015, July 20) Stephen Hawking and Yuri Milner announce \$100M initiative to seek ET. Sci Am.

Borucki, W.J., Koch, D., Basri, G., Batalha, N., Brown, T., Caldwell, D., Caldwell, J., Christensen-Dalsgaard, J., Cochran, W.D., DeVore, E., Dunham, E.W., Dupree, A.K., Gautier, T.N., Geary, J.C., Gilliland, R., Gould, R., Howell, S.B., Jenkins, J., Kondo, Y., Latham, D.W., Marcy, G.W., Mejbom, S., Kjeldsen, H., Lissauer, J.J., Monet, D.G., Morrison, D., Sasselov, D., Tarter, J., Boss, A., Brownlee, D. Owen, T., Buzasi, D., Charbonneau, D., Doyle, L., Fortney, J., Ford, E.B., Holman, M.J., Seager, S., Steffen, J.H., Welsh, W.F., Rowe, J., Anderson, H., Buchhave, L., Ciardi, D. Walkowicz, L., Sherry, W., Horch, E., Isaacson, H., Everett, M.E., Fisher, D., Torres, G., Johnson, J.A., Endl, M., MacQueen, P., Bryson, S.T., Dotson, J., Haas, M., Kolodziejczak, J., Van Cleve, J., Chandrasekaran, H., Twicken, J.D., Quintana, E.V., Clarke, B.D., Allen, C., Lie, J., Wu, H., Tenembaum, P., Verner, E., Bruhweiler, F., Barnes, J., and Prsa, A. (2010) Kepler planet-detection mission: introduction and first results. Science 327:977-980.

Brack, A. (2005) From the origin of life on Earth to life in the Universe. In Lectures in Astrobiology, Vol. 1, edited by $\mathrm{M}$. Gargaud, Springer, Berlin, pp 3-21.

Branscomb, E. and Russell, M.J. (2013) Turnstiles and bifurcators: the disequilibrium converting engines that put metabolism on the road. Biochim Biophys Acta 1827: 62-78.

Burchell, M.J., Mann, J.R., and Bunch, A.W. (2004) Survival of bacteria and spores under extreme pressures. Mon Not $R$ Astron Soc 352:1273-1278.

Bürger, R. and Lynch, M. (1995) Evolution and extinction in a changing environment: a quantitative-genetic analysis. Evolution 49:151-163.

Burrows, A.S. (2014) Highlights in the study of exoplanet atmospheres. Nature 513:345-352.

Chopra, A. and Lineweaver, C.H. (2016) The case for a Gaian bottleneck: the biology of habitability. Astrobiology 16:7-22.

Cleland, C.E. and Copley, S.D. (2005) The possibility of alternative microbial life on Earth. International Journal of Astrobiology 4:165-173.

Cocconi, G. and Morrison, P. (1959) Searching for interstellar communications. Nature 184:844-846.

Cockell, C.S., Bush, T., Bryce, C., Direito, S., Fox-Powell, M., Harrison, J.P., Lammer, H., Landenmark, H., Martin-Torres, J., Nicholson, N., Noack, L., O’Malley-James, J., Payler, S.J., Rushby, A., Samuels, T., Schwendner, P., Wadsworth, J., and Zorzano, M.P. (2016) Habitability: a review. Astrobiology 16:89-117.

Colwell, R.K. (2009) Biodiversity: concepts, patterns and measurement. In The Princeton Guide to Ecology, edited by S.A. Levin, Princeton University Press, Princeton, NJ, pp 257-263.

Colwell, R.K. and Coddington, J.A. (1994) Estimating terrestrial biodiversity through extrapolation. Philos Trans $R$ Soc Lond B Biol Sci 345:101-118.

Cowan, J.J. and Sneden, C. (2006) Heavy element synthesis in the oldest stars and early universe. Nature 440:1151-1156.

Crossfield, I.J.M. (2015) Observations of exoplanet atmospheres. Publ Astron Soc Pac 127:941-960.
Crowe, M.J. (2011) The Extraterrestrial Life Debate, 17501900, Dover Publications, Mineola, NY.

Darling, D. and Schulze-Makuch, D. (2016) The Extraterrestrial Encyclopedia, First Edition Design Publishing, Sarasota, FL.

Davies, P.C., Benner, S.A., Cleland, C.E., Lineweaver, C.H., McKay, C.P., and Wolfe-Simon, F. (2009) Signatures of the shadow biosphere. Astrobiology 9:241-291.

Davila, A.F. and McKay, C.P. (2014) Chance and necessity in biochemistry: implications for the search of extraterrestrial biomarkers in Earth-like environments. Astrobiology 14:534540.

de Fontenelle, B.B. (1686) Entretiens sur la Pluralité des Mondes, Nouvelle Edition augmentée (1701), Pierre Mortier, Librairie fur le Vygen-Dam, Amsterdam.

Deroo, P., Swain, M.R., and Green, O. (2012) Spectroscopy of exoplanet atmospheres with the FINESSE Explorer mission. Proc SPIE 8442, doi:10.1117/12.925236.

Des Marais, D.J. (1999) Astrobiology: exploring the origins, evolution, and distribution of life in the Universe. Annu Rev Ecol Syst 30:397-420.

Des Marais, D.J., Nuth, J.A., III, Allamandola, L.J., Boss, A.P., Farmer, J.D., Hoehler, T.M., Jakosky, B.M., Meadows, V.S., Pohorille, A., Runnegar, B., and Spormann, A.M. (2008) The NASA Astrobiology Roadmap. Astrobiology 8:715-730.

Descartes, R. (1644) Principia Philosophiae, apud Ludovicum Elzevirum, Ghent University, Belgium.

Dick, S.J. (1984) Plurality of Worlds: The Origins of the Extraterrestrial Life Debate from Democritus to Kant, Cambridge University Press, Cambridge, UK.

Dietrich, L.E.P, Michael, M., and Newman, D.K. (2006) The co-evolution of life and Earth. Curr Biol 16:pR395-pR400.

Domagal-Goldman, S.D., Meadows, V.S., Claire, M.W., and Kasting, J.F. (2011) Using biogenic sulfur gases as remotely detectable biosignatures on anoxic planets. Astrobiology 11:419-441.

Doyle, L.R., McCowan, B., Johnston, S., and Hanser, S.F. (2011) Information theory, animal communication, and the search for extraterrestrial intelligence. Acta Astronaut, SETI Special Edition, 68:406-417.

Drake, F. (2011) The search for extra-terrestrial intelligence. Philos Trans A Math Phys Eng Sci 369:633-643.

Dyson, F.J. (1999) Origins of Life, $2^{\text {nd }}$ ed., Cambridge University Press, Cambridge, UK.

Eliott, J. (2011a) A human language corpus for interstellar message construction. Acta Astronaut, SETI Special Edition, $68: 418-424$.

Eliott, J. (2011b) A semantic "engine" for universal translation. Acta Astronaut, SETI Special Edition, 68:435-440.

Eliott, J. (2011c) A post-detection decipherment strategy. Acta Astronaut, SETI Special Edition, 68:441-444.

Fabbro, F., Aglioti, S.M., Bergamasco, M., Clarici, A., and Panksepp, J. (2015) Evolutionary aspects of self- and world consciousness in vertebrates. Front Hum Neurosci 9, doi:10.3389/fnhum.2015.00157.

Fitz, D., Reiner, H., and Rode, B.M. (2007) Chemical evolution toward the origin of life. Pure Appl Chem 79:2101-2117.

Flammarion, C. (1872) La Pluralité des Mondes Habités, Didier, Paris.

Forgan, D.H. (2014) Can collimated extraterrestrial signals be intercepted? J Br Interplanet Soc 67:232-236.

Gaidos, E., Anderson, D.R., Lepine, S., Colon, K.D., Maravelias, G., Narita, N., Chang, E., Beyer, J., Fukui, A., Armstrong, J.D., Zezas, A., Fulton, B.J., Mann, A.W., West, 
R.G., and Faedi, F. (2014) Trawling for transits in a sea of noise: a search for exoplanets by analysis of WASP optical light curves and follow-up (SEAWOLF). Mon Not $R$ Astron Soc 437:3133-3143.

Garber, S.J. (1999) Searching for good science: the cancellation of NASA's SETI Program. J Br Interplanet Soc 52:3-12.

Gaston, K.J. (2000) Global patterns in biodiversity. Nature 405:220-227.

Geary, D.C. (2005) The Origin of the Mind: Evolution of Brain, Cognition, and General Intelligence, $1^{\text {st }}$ ed., American Psychological Association, Washington, DC.

Gibb, B.C. (2013) Life, the Universe and nomenclature. Nat Chem 5, doi:10.1038/nchem.1530.

Gladman, B., Dones, L., Levison, H.F., and Burns, J.A. (2005) Impact seeding and reseeding in the inner Solar System. Astrobiology 5:483-496.

Golding, S.D. and Glikson, M. (2011) Earliest Life on Earth: Habitats, Environments and Methods of Detection, Springer, Dordrecht, the Netherlands.

Gould, S.J. (1998) Evolution and Extinction, Audio Scholar, Mendocino, CA.

Graham, S.L., Snir, M., and Patterson, C.A., editors. (2005) The Future of Supercomputing, The National Academies Press, Washington, DC.

Grazier, K.R. (2016) Jupiter: cosmic Jekyll and Hyde. Astrobiology 16:23-38.

Grenfell, J.L., Rauer, H., Selsis, F., Kaltenegger, L., Beichman, C., Danchi, W., Eiroa, C., Fridlund, M., Henning, T., Herbst, T., Lammer, H., Léger, A., Lisaeu, R., Lunine, J., Paresce, F., Penny, A., Quirrenbach, A., Röttgering, H., Schneider, J., Stam, D., Tinetti, G., and White, G.J. (2010) Co-evolution of atmospheres, life, and climate. Astrobiology 10:77-88.

Grinspoon, D.H. (2012) Assessing the Anthropocene in the context of comparative planetology [abstract \#P14A-02]. In American Geophysical Union, Fall Meeting 2012, American Geophysical Union, Washington, DC.

Gulkis, S., Olsen, E.T., and Tarter, J.C. (1981) A bimodal search strategy for SETI. In Strategies for the Search for Life in the Universe, edited by M.D. Papagiannis, D. Reidel Publishing Co., Dordrecht, the Netherlands, pp 93-105.

Harp, G.R., Richards, J., Shostak, S., Tarter, J.C., Vakoch, D.A., and Munson, C. (2015) Radio SETI observations of the anomalous star KIC 8462852. arXiv:1511.01606

Hatzes, A.P. (2014) The detection of Earth-mass planets around active stars: the mass of Kepler-78B. Astron Astrophys 568, doi:10.1051/0004-6361/201424025.

Heath, M.J., Doyle, L.R., Joshi, M.J., and Haberle, R. (1998) Habitability of planets around red dwarf stars. Orig Life Evol Biosph 29:405-424.

Heinecke, A., Eckhardt, W., Horsch, M., and Bungartz, H.-J. (2015) Supercomputing for Molecular Dynamics Simulations: Handling Multi-Trillion Particles in Nanofluidics, Springer Briefs in Computer Science, Springer International Publishing, Cham, Switzerland.

Heller, R. and Pudritz, R.E. (2016) The search for extraterrestrial intelligence in Earth's solar transit zone. Astrobiology $16: 259-270$

Heller, R., Leconte, J., and Barnes, R. (2011) Tidal obliquity evolution of potentially habitable planets. Astron Astrophys 528, doi:10.1051/0004-6361/201015809.

Horner, J., Waltham, D., and Koch, F.E. (2013) The role of Jupiter in driving Earth's orbital evolution. In Proceedings of the $13^{\text {th }}$ Australian Space Science Conference, Sept. 30-Oct. 2, 2013.
Houri-Ze'evi, L., Korem, Y., Sheftel, H., Faigenbloom, L., Toker, I.A., Dagan, Y., Awad, L., Degani, L., Alon, U., and Rechavi, O. (2016) A tunable mechanism determines the duration of the transgenerational small RNA inheritance in $C$. elegans. Cell 165:88-99.

Ilhan, H. and Linscott, I. (2011) Using fractal derivatives as "degree of symmetry" to characterize natural shapes. Acta Astronaut, SETI Special Edition, 68:425-434.

Imari Walker, S. (2014) Transition from abiotic to biotic: is there an algorithm for it? In An Evolutionary Approach, edited by V. Kolb, CRC Press, Boca Raton, FL, pp 371-398.

Irwin, N.I. and Schulze-Makuch, D. (2001) Assessing the plausibility of life on other worlds. Astrobiology 1:143-160.

Irwin, N.I., Méndez, A., Fairén, A.G., and Schulze-Makuch, D. (2014) Assessing the possibility of biological complexity on other worlds, with an estimate of the occurrence of complex life in the Milky Way Galaxy. Challenges 5:159-174.

Jerison, H.J. and Jerison, I. (2013) Intelligence and Evolutionary Biology, NATO ASI Series G, Ecological Sciences, Vol. 17. Springler-Verlag, Berlin.

Kant, I. (2009) Universal Natural History and Theory of the Heavens, Translated by I.C. Johnston, Richer Resources Publications, Arlington, VA. (Original work published in 1755)

Kasting, J.F. (1993) Earth's earliest atmosphere. Science 259:920-926.

Keller, G. and Kerr, A. (2014) Volcanism, Impacts, and Mass Extinctions: Causes and Effects, GSA Special Paper 505, Geological Society of America, Boulder, CO.

Kipping, D.M. and Teachey, A. (2016) A cloaking device for transiting planets. Mon Not R Astron Soc 458, doi:10.1093/ mnras/stw672.

Knoll, A.H. (2009) The co-evolution of life and environments. Rendiconti Lincei Scienze Fisiche e Naturali 20:301-306.

Kolb, V. (2014) Astrobiology: An Evolutionary Approach, CRC Press, Boca Raton, FL.

Kooijman, S.A.L.M. (2004) On the co-evolution of life and its environment. In Scientists Debate Gaia: The Next Century, edited by S.H. Schneider, J.R. Miller, E. Crist, and P.J. Boston, MIT Press Scholarship Online, Cambridge, MA, doi:10.7551/mitpress/9780262194983.003.0032.

Kopparapu, R., Ravi, K., Ramirez, R., Kasting, J.F., Eymet, V., Robinson, T.D., Mahadevan, S., Terrien, R., Ryan, C., DomagalGoldman, S., Meadows, V., and Deshpande, R. (2013) Habitable zones around main sequence stars: new estimates. Astrophys $J$ 765, doi:10.1088/0004-637X/765/2/131.

Krissansen-Totton, J., Bergsman, D.S., and Catling, D.C. (2016) On detecting biospheres from chemical thermodynamic disequilibrium in planetary atmospheres. Astrobiology 16:39-67.

Krubitzer, L. (2014) Lessons from evolution. In The Future of the Brain: Essays by the World's Leading Neuroscientists, edited by G. Marcus and J. Freeman, Princeton University Press, Princeton, NJ, pp 186-193.

Lai, S., Safaei, J., and Pelech, S. (2016) Evolutionary ancestry of eukaryotic protein kinases and choline kinases. $J$ Biol Chem 291:5199-5205.

Laskar, J., Joutel, F., and Robutel, P. (1993) Stabilization of the Earth's obliquity by the Moon. Nature 361:615-617.

Lazcano, A. and Miller, S.L. (1996) The origin and early evolution of life: prebiotic chemistry, the pre-RNA world, and time. Cell 85:793-798.

Lehman, J. and Miikkulainen, R. (2015) Extinction events can accelerate evolution. PLoS One 10, doi:10.1371/journal.pone .0132886 . 
Lissauer, J.J., Barnes, J.W., and Chambers, J.E. (2011) Obliquity variations of a moonless Earth. Icarus 217:77-87.

Locey, K.J. and Lennon, J.T. (2016) Scaling laws predict global microbial diversity. Proc Natl Acad Sci USA 113:5970-5975.

Lowell, P. (1906) Mars and Its Canals, Macmillan Company, New York.

Lynch, M. and Lande, R. (1993) Evolution and extinction in response to environmental change. In Biotic Interactions and Global Change, edited by J.G. Kingsolver and R.B. Huey, Sinauer, Sunderland, MA.

Lyons, T.W., Reinhard, C.T., and Planavsky, N.J. (2014) The rise of oxygen in Earth's early ocean and atmosphere. Nature 506:307-315.

Maccone, C. (2012) Mathematical SETI: Statistics, Signal Processing, Space Missions, Springer-Verlag, Berlin.

Macintosh, B., Graham, J.R., Barman, T., De Rosa, R.J., Konopacky, Q., Marley, M.S., Marois, C., Nielsen, E.L, Pueyo, L., Rajan, A., Rameau, J., Saumon, D., Wang, J.J., Ammons, M., Arriaga, P., Artigau, E., Beckwith, S., Brewster, J., Bruzzone, S., Bulger, J., Burningham, B., Burrow, A.S., Chen, C., Chiang, E., Chilcote, J.K., Dawson, R.I., Dong, R., Doyon, R., Draper, Z.H., Duchêne, G., Esposito, T.M., Fabrycky, D., Fitzgerald, M.P., Follette, K.B., Fortney, J.J., Gerard, B., Goodsell, S., Greenbaum, A.Z., Hibon, P., Hinkley, S., Cotton, T.H., Hung, L.-W., Ingraham, P., Johnson-Groh, M., Kalas, P., Lafreniere, D., Larkin, J.E., Lee, J., Line, M., Long, D., Maire, J., Marchis, F., Matthews, B.C., Max, C.E., Metchev, S., Millar-Blanchaer, M.A., Mittal, T., Morley, C.V., Morzinskim, K.M., Murray-Clay, R., Oppenheimer, R., Palmer, D.W., Patel, R., Patience, J., Perrin, M.D., Poyneer, L.A., Rafikov, R.R., Rantakyrö, F.T., Rice, E., Rojo, P., Rudy, A.R., Ruffio, J.-B., Ruiz, M.T., Sadakuni, N., Saddlemyer, L., Salama, M., Savransky, D., Schneider, A.C., Sivaramakrishnan, A., Song, I., Soummer, R., Thomas, S., Vasisht, G., Wallace, J.K., Ward-Duong, K., Wiktorowicz, S.J., Wollff, S.G., and Zuckerman, B. (2015) Discovery and spectroscopy of the young jovian planet 51 Eri $\mathrm{b}$ with the Gemini Planet Imager. Science 350:64-67.

Mandelbrot, B.B. (1982) The Fractal Geometry of Nature, W.H. Freeman and Company, San Francisco.

Mandell, A.V. (2008) Expanding and improving the search for habitable worlds. In New Horizons in Astronomy: Frank N. Bash Symposium 2007, ASP Conference Series Vol. 393, Astronomical Society of the Pacific, San Francisco, pp 19-34.

Margulis, L. (1996) Archaeal-eubacterial mergers in the origin of Eukarya: phylogenetic classification of life. Proc Natl Acad Sci USA 93:1071-1076.

Martin, W., Baross, J., Kelley, D., and Russel, M. (2008) Hydrothermal vents and the origin of life. Nat Rev 6:805-814.

McCollom, T.M. (2013) Miller-Urey and beyond: what have we learned about prebiotic organic synthesis reactions in the past 60 years? Annu Rev Earth Planet Sci 41:207-229.

McGinley, M. (2014) Species richness. In The Encyclopedia of Earth (EoE). Retrieved from http://www.eoearth.org/view/ article/156216

McKay, C.P. (2004) What is life-and how do we search for it in other worlds? PLoS Biol 2, doi:10.1371/journal.pbio .0020302 .

Melosh, J. (1996) Impact Cratering: A Geologic Process, Oxford Monographs on Geology and Geophysics no. 11, Oxford University Press, New York.

Monod, J. (1972) Chance and Necessity: An Essay on the Natural Philosophy of Modern Biology, Vintage Books, New York.
Mora, C., Tittensor, D.P., Adl, S., Simpson, A.G.B., and Worm, B. (2011) How many species are there on Earth and in the ocean? PLoS Biol 9, doi:10.1371/journal.pbio.1001127.

Musso, P. (2012) The problem of active SETI: an overview. Acta Astronaut 78:43-54.

Napier, W.M. (2004) A mechanism for interstellar panspermia. Mon Not $R$ Astron Soc 348:46-51.

Neal, M. (2014) Preparing for extraterrestrial contact. Risk Management 16:63-87.

Nealson, K.H. and Conrad, P.G. (1999) Life: past, present and future. Philos Trans R Soc Lond B Biol Sci 354:19231939.

Nolfi, S. and Parisi, D. (1996) Learning to adapt to changing environments in evolving neural networks. Adapt Behav 5:75-98.

Ollongren, A. and Vakoch, D.A. (2011) Large-size message construction for ETI: logical existence expressed in Lingua Cosmica. In SETI Science and Technology Review Meeting, $62^{\text {nd }}$ International Astronautical Congress, Cape Town, South Africa.

O’Malley-James, J.T., Cockell, C.S., Greaves, J.S., and Raven, J.A. (2014) Swansong biospheres II: The final signs of life on terrestrial planets near the end of their habitable lifetimes. International Journal of Astrobiology 13:229-243.

Owen, T. (2008) The contributions of comets to planets, atmospheres, and life: insights from Cassini-Huygens, Galileo, Giotto, and inner planet missions. Origin and Early Evolution of Comet Nuclei: Workshop Honouring Johannes Geiss on the Occasion of His $80^{\text {th }}$ Birthday, Space sciences series of ISSI, Vol. 28, Springer, New York, pp 301-316.

Pascal, R., Boiteau, L., Forterre, P., Gargaud, M., Lazcano, A., López-García, P., Moreira, D., Maurel, M.-C., Peretó, J., Prieur, D., and Reisse, J. (2006) Prebiotic chemistry-biochemistry-emergence of life (4.4-2 Ga). Earth Moon Planets 98:153-203.

Perlovsky, L.I. (2006) Toward physics of the mind: concepts, emotions, consciousness, and symbols. Phys Life Rev 3:23-55.

Petigura, E.A., Howard, A.W., and Marcy, G.W. (2013) Prevalence of Earth-size planets orbiting Sun-like stars. Proc Natl Acad Sci USA 110:19273-19278.

Pfeifer, R. and Gómez, G. (2006) Morphological computation-connecting brain, body, and environment. In Creating Brain-Like Intelligence, Lecture Notes in Computer Science 5436, edited by B. Sendhoff, Springer, Berlin, pp 66-83.

Pigliucci, M. and Müller, G.B., editors. (2010) Evolution, the Extended Synthesis, MIT Press, Cambridge, MA.

Pilcher, C.B. (2004) Biosignatures of early Earths. Astrobiology 3:471-486.

Popa, R. (2004) Between Necessity and Probability: Searching for the Definition and Origin of Life, Springer, Berlin.

Pringle, J.W.S. (1951) On the parallel between learning and evolution. Behaviour 3:174-214.

Pross, A. (2012) What Is Life? How Chemistry Becomes Biology, Oxford University Press, Oxford, UK.

Pross, A. and Pascal, R. (2013) The origin of life: what we know, what we can know, and what we will never know. Open Biology 3, doi:10.1098/rsob.120190.

Quintana, E.V., Barclay, T., Raumond, S.N., Rowe, J.F., Bolmont, E., Caldwell, D.A., Howell, S.B., Kane, S.R., Huber, D., Crepp, J.R., Lissauer, J.J., Ciardi, D.R., Coughlin, J.L., Everett, M.E., Henze, C.E., Horch, E., Isaacson, H., Ford, E.B., Adams, F.C., Still, M., Hunter, R.C., Quarles, B., and Selsis, F. (2014) An Earth-sized planet in the habitable zone of a cool star. Science 344:277-280. 
Rao, R.P.N., Stocco, A., Bryan, M., Sarma, D., and Youngquist, T.M. (2014) A direct brain-to-brain interface in humans. PLoS One 9, doi:10.1371/journal.pone.0111332.

Raup, D.M. (1999) The role of extinction in evolution. Proc Natl Acad Sci USA 91:6758-6763.

Rose, C. and Wright, G. (2004) Inscribed matter as an energyefficient means of communication with an extraterrestrial civilization. Nature 431:47-49.

Rosenfeld, S. (2011) Mathematical descriptions of biochemical networks: stability, stochasticity, evolution. Prog Biophys Mol Biol 106:400-409.

Roth, G. (2013) The Long Evolution of Brains and Minds, Springer, Dordrecht, the Netherlands.

Ruiz-Mirazo, K., Brionest, C., and de la Escosura, A. (2013) Prebiotic systems chemistry: new perspectives on the origins of life. Chem Rev 114:285-366.

Russell, M.J., Nitschke, W., and Branscomb, E. (2013) The inevitable journey to being. Philos Trans $R$ Soc Lond B Biol Sci 368, doi:10.1098/rstb.2012.0254.

Sagan, C. (1975) Communication with Extraterrestrial Intelligence, MIT Press, Cambridge, MA.

Sasselov, D. (2012) The Life of Super-Earths: How the Hunt for Alien Worlds and Artificial Cells Will Revolutionize Life on our Planet, Basic Books, New York.

Schrey, A.W., Richards, C.L., Meller, V., Sollars, V., and Ruden, D.M. (2012) The role of epigenetics in evolution: the extended synthesis. Genet Res Int 2012, doi:10.1155/2012/286164.

Schuch, H.P. (2011) Project Ozma: the birth of observational SETI. In Searching for Extraterrestrial Intelligence, The Frontiers Collection, Springer, Berlin, pp 13-19.

Schuetz, M., Vakoch, D.A., Shostak, S., and Richards, J. (2015) Optical SETI observations of the anomalous star KIC 8462852. arXiv: 1512.02388

Schulkin, J. (2014) Cognitive Adaptation: A Pragmatist Perspective, Cambridge University Press, Cambridge, UK.

Schulze-Makuch, D. and Irwin, L.N. (2001) Lessons from the history of life on Earth. In Life in the Universe, edited by D. Schulze-Makuch and L.N. Irwin, Advances in Astrobiology and Biogeophysics, Springer, Berlin, pp 25-41.

Schulze-Makuch, D., Irwin, L.N., and Fairén, A.G. (2013) Drastic environmental change and its effects on a planetary biosphere. Icarus 225:775-780.

Schwartz, R.N. and Townes, C.H. (1961) Interstellar and interplanetary communication by optical masers. Nature 190:205208.

Schwartzman, D.W. (2004) Does life drive disequilibrium in the biosphere? In Scientists Debate Gaia: The Next Century, edited by S.H. Schneider, J.R. Miller, E. Crist, and P.J. Boston, MIT Press, Cambridge, MA, doi:10.7551/mitpress/ 9780262194983.003.0013.

Schwieterman, E.W., Meadows, V.S., Domagal-Goldman, S.D., Deming, D., Arney, G.N., Luger, R., Harman, C.E., Misra, A., and Barnes, R. (2016) Identifying planetary biosignature impostors: spectral features of $\mathrm{CO}$ and $\mathrm{O}_{4}$ resulting from abiotic $\mathrm{O}_{2} / \mathrm{O}_{3}$ production. Astrophys $J$ 819, doi:10.3847/ 2041-8205/819/1/L13.

Seager, S. (2013) Exoplanet habitability. Science 340:577-581.

Seager, S. (2014) The future of spectroscopic life detection on exoplanets. Proc Natl Acad Sci USA 11:12634-12640.

Seager, S. and Deming, D. (2010) Exoplanet atmospheres. Annu Rev Astron Astrophys 48:631-672.

Seckbach, J., editor. (2006) Life as We Know It, Cellular Origin and Life in Extreme Habitats and Astrobiology, Vol. 10, Springer, Dordrecht, the Netherlands.
Seckbach, J., Chela-Flores, J., Owen, T., and Raulin, F., editors. (2004) Life in the Universe: From the Miller Experiment to the Search for Life on Other Worlds, Cellular Origin and Life in Extreme Habitats and Astrobiology, Vol. 7, Springer, Dordrecht, the Netherlands.

Segura, A., Kasting, J.F., Meadows, V., Cohen, M., Scalo, J., Crisp, D., Butler, R.A.H., and Tinetti, G. (2005) Biosignatures from Earth-like planets around M dwarfs. Astrobiology 5:706-725.

Seifer, M.J. (1996) Martian fever (1895-1896). In Wizard: The Life and Times of Nikola Tesla: Biography of a Genius, Carol Publishing Group, Secaucus, NJ.

Shapiro, R. (2006) Small molecule interactions were central to the origin of life. $Q$ Rev Biol 81:105-125.

Shostak, S. (1998) Sharing the Universe: Perspectives on Extraterrestrial Life, Berkeley Hills Book, Berkeley, CA.

Shostak, S. (2011a) Short-pulse SETI. Acta Astronaut, SETI Special Edition, 68:362-365.

Shostak, S. (2011b) Limits on interstellar messages. Acta Astronaut, SETI Special Edition, 68:366-371.

Smith, R.E. and Cribbs, H.B., III, editors. (1994) Evolutionary Computation, MIT Press, Cambridge, MA.

Spohn, T. (1991) Mantle differentiation and thermal evolution of Mars, Mercury, and Venus. Icarus 90:222-236.

Stern, R.J. (2015) Is plate tectonics needed to evolve technological species on exoplanets? Geoscience Frontiers 7:573-580.

Strausfeld, N.J. and Hirth, F. (2015) Introduction to "Homology and convergence in nervous system evolution." Philos Trans $R$ Soc Lond B Biol Sci 317, doi:10.1098/rstb.2015.0034.

Summons, R.E., Amend, J.P., Bish, D., Buik, R., Cody, G.D., Des Marais, D.J., Dromart G., Eigenbrode, J.L., Knoll, A.H., and Sumner, D.Y. (2011) Preservation of martian organic and environmental records: final report of the Mars Biosignature Working Group. Astrobiology 11:157-181.

Swendenborg, E. (1758) Life on Other Planets, $1^{\text {st }}$ ed., Swedenborg Foundation, West Chester, PA.

Tarter, J.C. (1979) The Cosmic Haystack, Harlan Smith Working Group on SETI of the National Academy of Sciences Astronomy and Astrophysics for the 1980s Survey Committee.

Tarter, J.C. (1983) SETI Program. Letter to Science 22:359.

Tarter, J.C. (2001a) The search for extraterrestrial intelligence (SETI). Апnu Rev Astron Astrophys 39:511-548.

Tarter J.C. (2001b) What is SETI? Ann NY Acad Sci 950:269_ 275.

Tarter, J.C. (2004) Astrobiology and SETI. New Astronomy Reviews 48:1543-1549.

Tarter, J.C. (2007) The evolution of life in the Universe: are we alone? Proceedings of the International Astronomical Union 2:14-29.

Tarter, J.C. and Chyba, C.F. (1999) Is there life elsewhere in the Universe? Sci Am 281:80-85.

Tarter, J.C., Backus, P., Mancinelli, R., Aurnou, J., Backman, D., Basri, G., Boss, A., Clarke, A., Deming, D., Doyle, L., Feigelson, E., Freund, F., Grinspoon, D., Haberle, R., Hauck, S., Heath, M., Henry, T., Hollingsworth, J., Joshi, M., Jura, M., Kilston, S., Laughlin, G., Liu, E., Meikle, M., Reid, I., Rothschild, L., Scalo, J., Segura, A., Tang, C., Tiedje, J., Turnbull, M., Walkowicz, L., Weber, A., and Young, R. (2007) A reappraisal of the habitability of planets around $M$ dwarf stars. Astrobiology 7:30-65.

Turnbull, M.C. and Tarter, J.C. (2003) Target selection for SETI. I. A catalog of nearby habitable stellar systems. Astrophys J Suppl Ser 145:181-198. 
Vakoch, D.A., editor. (2011a) Communication with Extraterrestrial Intelligence, State University of New York Press, Albany, NY.

Vakoch, D.A. (2011b) A taxonomic approach to communicating maxims in interstellar messages. Acta Astronaut, SETI Special Edition, 68:500-511.

Vakoch, D.A. (2011c) A narratological approach to interpreting and designing interstellar messages. Acta Astronaut, SETI Special Edition, 68:520-534.

Vakoch, D.A., editor. (2014) Between Worlds: The Art and Science of Interstellar Message Composition, MIT Press, Cambridge, MA.

Vakoch, D.A. and Dowd, M.F. (2015) The Drake Equation: Estimating the Prevalence of Extraterrestrial Life through the Ages, Cambridge Astrobiology Series Book 8, Cambridge University Press, Cambridge, UK.

Vakoch, D.A. and Harrison, A.H., editors. (2011) Civilization beyond Earth: Extraterrestrial Life and Society, Berghahn Books, New York.

Vakoch, D.A. and Matessa, M. (2011) An algorithmic approach to communicating reciprocal altruism in interstellar messages: drawing analogies between social and astrophysical phenomena. Acta Astronaut, SETI Special Edition, 68:459-475.

Valiant, L. (2013) Probably Approximately Correct: Nature's Algorithms for Learning and Prospering in a Complex World, Basic Books, New York.

Voltaire, F.-M.A. (2002) Micromégas and Other Short Fictions, translated by T. Cuffe and H.T. Mason, Penguin Classics, New York. (Original work published in 1752)

Wagner, A. (2007) Robustness and Evolvability in Living Systems, Princeton University Press, Princeton, NJ.

Wagner, G.P. and Draghi, J. (2010) Evolution of evolvability. In Evolution: The Extended Synthesis, edited by M. Pigliucci and G.B. Müller, MIT Press, Cambridge, MA, pp 218-228.

Wagner, G.P., Pavlicev, M., and Cheverud, J.M. (2007) The road to modularity. Nat Rev Genet 8:921-931.

Walker, S.I. (2014) Top-down causation and the rise of information in the emergence of life. Information 5:424-439.

Ward, P.D. and Benner, S.A. (2007) Alien biochemistries. In Planets and Life: The Emerging Science of Astrobiology, edited by W.T. Sullivan III and J.A. Baross, Cambridge University Press, Cambridge, UK, pp 537-544.

Ward, P.D. and Brownlee, D. (2003) Rare Earth: Why Complex Life is Uncommon in the Universe, Copernicus Books, New York.

Waters, C.N., Zalasiewicz, J., Summerhayes, C., Barnosky, A.D., Poirier, C., Galuszka, A., Cearreta, A., Edgeworth, M., Ellis, E.C., Ellis, M., Jeandel, C., Leindfelder, R., McNeill, J.R., deB. Richter, D., Steffen, W., Syvitski, J., Vids, D., Wagreich, M., Williams, M., Grinevald, J., Odada, E., Oreskes, N., and Wolfe, A.P. (2016) The Anthropocene is functionally and stratigraphically distinct from the Holocene. Science 351, doi:10.1126/science.aad2622.
Watson, A.J. (1999) Coevolution of the Earth's environment and life: Goldilocks, Gaia and the anthropic principle. Geol Soc Spec Publ 150:75-88.

Watson, R.A. and Szathmáry, E. (2015) How can evolution learn? Trends Ecol Evol 31:147-157.

Wheeler, E. (2014) The "Wow" signal, Drake equation, and exoplanet considerations. J Br Interplanet Soc 67:412-417.

Witzany, G. (2015) Life is physics and chemistry and communication. Ann NY Acad Sci 1341:1-9.

Wolf, Y.I., Rogozin, I.B., Grishin, N.V., and Koonin, E.V. (2002) Genome trees and the tree of life. Trends Genet 18:472-479.

Wolpaw, J.R. and Winter Wolpaw, E., editors. (2012) BrainComputer Interfaces: Principles and Practice, Oxford University Press, Oxford, UK.

Worth, R.J., Sigurdsson, S., and House, C.H. (2013) Seeding life on the moons of the outer planets via lithopanspermia. Astrobiology 13:1155-1165.

Wright, S.A., Werthimer, D., Treffers, R.R., Maire, J., Marcy, G.W., Stone, R.P.S., Drake, F., Meyer, E., Dorval, P., and Siemon, A. (2014) A near-infrared SETI experiment: instrument overview. Proc SPIE 9147, doi:10.1117/12.2055616.

Yeomans, D.K. (2013) Near-Earth Objects: Finding Them Before They Find Us, Princeton University Press, Princeton, NJ.

Zackrisson, E., Calissendorff, P., González, J., Benson, A., Johansen, A., and Janson, M. (2016) Terrestrial planets across space and time. arXiv: 1602.00690

Ziman, J., editor. (2000) Technological Innovation as an Evolutionary Process, Cambridge University Press, Cambridge, UK.

Zuckerman, B. and Tarter, J.C. (1979) Is there anyone out there? Nature 281:528-529.

Address correspondence to: Dr. Nathalie A. Cabrol

SETI Institute Carl Sagan Center 189 N Bernardo Ave. \#200

Mountain View, CA 94043

E-mail: ncabrol@seti.org

Submitted 14 May 2016

Accepted 23 May 2016

$\begin{aligned} & \text { Abbreviations Used } \\ \mathrm{ET} & =\text { extraterrestrial life } \\ \mathrm{ETI} & =\text { extraterrestrial intelligence } \\ \mathrm{METI} & =\text { Messaging Extraterrestrial Intelligence } \\ \mathrm{NAI} & =\text { NASA Astrobiology Institute } \\ \mathrm{SETI} & =\text { Search for Extraterrestrial Intelligence }\end{aligned}$

